\title{
Testing tumors for microsatellite instability
}

\author{
A Ibert de la Chapelle \\ H uman Cancer G enetics Program, Comprehensive Cancer Center, The O hio State U niversity, Columbus, O hio, USA
}

\begin{abstract}
The methods for determining microsatellite instability in tumors are highly heterogeneous. R ecently a 5-marker panel of microsatellites was suggested for this purpose. In this review attention is drawn to the fact that microsatellite instability can be assessed by analyzing tumor D NA with a single marker, BAT-26, and that normal tissue DNA from the same individual needs to be analyzed only when an aberrant allele is seen in the tumor. Whilst this simple procedure does not distinguish between different types and degrees of instability, it should be sufficient for many purposes, such as screening colorectal cancers for mismatch repair deficiency.
\end{abstract}

Keywords: cancer; colorectal; microsatellite; instability; stability; hereditary; hereditary nonpolyposis colorectal cancer (H N PCC); replication error (RER ); microsatellite instability (MSI)

A recent report ${ }^{1}$ from the International Workshop on Microsatellite Instability and RER Phenotypes in Cancer Detection and Familial Predisposition, 8-9D ecember 1997, made recommendations regarding the testing and interpretation of microsatellite instability (MSI). In essence, a 'reference panel' of five microsatellites was recommended for future research in the field. Two important reservations were made. It was explicitly stated that the recommendations apply to colorectal cancer only. Furthermore, the text of the report states that other loci and panels may prove to be of equal utility.

$\mathrm{N}$ evertheless it is possible that the recommendations contained in the report may be taken too literally; that is, that M SI determinations may be viewed as deficient or unreliable if the recommended marker panel is not used. The purpose of this brief review is to draw attention to the fact that, as shown recently, a far easier and less costly, but not necessarily less efficacious, method of testing for MSI in colorectal cancer should be considered for some purposes.

Because M SI is a hallmark of tumors caused by the inactivation of mismatch repair genes, and because

Correspondence: A lbert de la Chapelle, MD, PhD, 646 $M$ edical R esearch Facility, 420 West $12^{\text {th }}$ A venue, Columbus, OH 43210, U SA. E-mail: delachapelle-1@medctr.osu.edu R eceived 18 January 1999; accepted 9 February 1999 some patients with $\mathrm{MSI}^{+}$tumors have germline mutations in mismatch repair genes, it is clinically relevant to screen for MSI followed by evaluation of the mismatch repair genes in the germlines of patients whose tumors are $\mathrm{MSI}^{+}$. Thus new cases and families with the hereditary non-polyposis colorectal cancer (HNPCC) syndrome will be detected and may benefit from highrisk clinical screening for cancer and precancerous conditions. It is now technically possible to screen for HNPCC among newly diagnosed colorectal cancer patients, and a significant, but not necessarily unique role in such screening belongs to $\mathrm{MSI}$ testing. There may be a need for thousands or tens of thousands of MSI tests for this purpose.

The markers BAT-26 and BAT-25 that are both contained in the recommended panel ${ }^{1}$ are single nucleotide tracts that show a single allele in the germline and normal somatic cells of most individuals. ${ }^{2,3}$ In BAT-26 this consists of a run of 26 adenosines, $(\mathrm{A})_{26}$. O ccasional individuals have 27,25 or $24 \mathrm{As}$ instead of 26. A s these individuals represent less than $5 \%$ of the population, the BAT-26 marker has been termed quasi-monomorphic. The polymorphic nucleotide run in marker BAT-25 mainly consists of $(\mathrm{T})_{7} \mathrm{~A}$ $(T)_{25}$. This allele is the most common; other alleles so far seen in normal individuals from the populations studied have either a loss of one nucleotide or an addition of one or two nucleotides. 
Both BAT-26 and BAT-25 are highly sensitive to MSI. In a first study of 160 colorectal cancer tumors and cell lines, the MSI status of 159 of these could be correctly determined by studying BAT-26 alone. ${ }^{4}$ This conclusion was made after comparing the results of BAT-26 with those of a combined panel of a minimum of 31 other microsatellite loci. In an extension of these studies, BAT-26 was analyzed in a total of 542 solid tumors from various organs. ${ }^{5}$ In 539/542 tumors BA T-26 identified microsatellite stability/instability concordant with the results from variable batteries of other microsatellite markers. In each case normal tissue was also studied. This allowed the quasi-monomorphic allele distribution of BAT-26 and the distribution of alleles in BAT-25 described above to be determined.

Recently, a prospective study of 509 consecutive, unselected, sporadic colorectal cancer patients was reported. ${ }^{6} \mathrm{U}$ sing a battery of other microsatellite markers, 446/509 tumors were classified as $\mathrm{M} \mathrm{SI}^{-}$whilst $63 / 509$ were $\mathrm{MSI}^{+}$. When BAT-26 was studied in all tumors, it showed abnormal alleles in six of the 446 tumors previously classified as $\mathrm{MSI}^{-}$and, further, showed abnormal alleles in 58 out of the 63 tumors previously classified as $\mathrm{MSI}^{+}$. A II 69 patients whose tumors were $\mathrm{MSI}$ positive for either the conventional markers or BAT-26 were subjected to mutational analyses by exon-by-exon sequencing of $\mathrm{MSH} 2$ and MLH 1 from non-tumor DNA. Ten patients were germline mutation positive (new cases of HNPCC); all ten were $\mathrm{MSI}$ positive both with conventional markers and BAT-26.

The evidence available so far lends itself to the suggestion that colorectal tumors can be M SI classified by studying BAT-26 only. N otably, it is not necessary to study normal tissue at first. I $n$ all patients whose tumors show alleles other than $(A)_{26}$, normal tissue should be studied as well so as to determine whether the change is acquired or germline. In this way misclassification due to rare aberrant germline alleles can be avoided. Such alleles have not yet been seen, or at least not identified as germline variants, ${ }^{5}$ but could well occur in other populations than the Caucasians who have been studied so far.

A vailable evidence shows that in MSI, BAT-26 always loses rather than gains $A$ s. So far, not a single tumor has been reported in which a gain of $A$ has occurred. The typical loss in BAT-26 comprises 5-15 nucleotides and, remarkably, there is no overlap between rare normal alleles with $(A)_{25}$ or $(A)_{24}$ and $M S I$ positive tumors with $(A)_{21}$ or less. $A$ gray zone of
(A ) ${ }_{22}$ or (A ) 2 $_{23}$ may exist. A ccording to the data by $Z$ hou et $\mathrm{al}^{7}$ these cases can be resolved into either $\mathrm{MSI}^{+}$or $\mathrm{MSI}^{-}$by studying BAT-25.

U ntil more data on the molecular background of MSI become available it is certainly recommendable to study many markers for various research purposes and the 5-marker panel recommended ${ }^{1}$ is a useful one, eg for comparisons between laboratories. My proposal is meant for the practical situation where colorectal, and perhaps other, tumors need to be tested for M SI for the purpose of evaluating the patient for HNPCC. MSI screened for in this way (a single marker studied in tumor tissue only) should be sufficiently easy and sensitive for clinical studies of both high and low-risk patients. Together with data on family history and age of onset this test provides a reasonable means of prescreening for HNPCC.

\section{Acknowledgements}

The author's research is supported by grants CA 67941 and CA 16058 from the National Institutes of H ealth.

\section{References}

1 Boland CR, Thibodeau SN, Hamilton SR et al: The International Workshop on Microsatellite Instability and RER Phenotypes in Cancer D etection and Familial Predisposition, summary and recommendations. Cancer R es 1998; 58: $5248-5257$.

2 Papadopoulos N, Nicolaides NC, Liu B et al: M utation of GTBP in genetically unstable cells. Science 1995; 268: 1915-1917.

3 D ietmaier W, Wallinger S, B ocker T, Kullmann F, Fishel R, $R$ üschoff J : D iagnostic microsatellite instability: D efinition and correlation with mismatch repair protein expression. Cancer Res 1997; 57: 4749-4756.

4 Hoang J M, Cottu PH, Thuille B, Salmon RJ, Thomas G, $H$ amelin $R$ : BAT-26, an indicator of the replication error phenotype in colorectal cancers and cell lines. Cancer Res 1997; 57: 300-303.

5 Z hou X - P, H oang J-M, Li Y -J et al: Determination of the replication error phenotype in human tumors without the requirement for matching normal DNA by analysis of mononucleotide repeat microsatellites. G enes Chrom Cancer 1998; 221: 101-107.

6 A altonen $L A$, Salovaara $R, K$ risto $P$ et al: Incidence of hereditary nonpolyposis colorectal cancer and the feasibility of molecular screening for the disease. New Engl J Med 1998; 338: 1481-1487.

7 Zhou X-P, Hoang J-M, Cottu P, Thomas G, Hamelin R: A llelic profiles of mononucleotide repeat microsatellites in control individuals and in colorectal tumors with and without replication errors. O ncogene 1997; 15: 1713-1718. 